\title{
On the relationship between cosmic rays detected by the KACST muon detector and solar wind speed and sunspot number
}

\author{
Maghrabi A.H ${ }^{1}$ \\ National Centre for Applied Physics, King Abdulaziz City for Science and Technology, \\ Address, P.O.Box 6086Riyadh 11442, Saudi Arabia. \\ Email:amaghrabi@kacst.edu.sa

\section{Al Dajani H.M} \\ National Centre for Applied Physics, King Abdulaziz City for Science and Technology, \\ Address, P.O.Box 6086Riyadh 11442, Saudi Arabia. \\ Email:haldajani@kacst.edu.sa
}

\begin{abstract}
Solar disturbances modulate cosmic rays on different time scales. Studying cosmic ray variation is an important subject that attracts scientists from different disciplines. In this study, correlation analyses between cosmic ray muons and both the solar wind speed and sunspot number for the period between 2002 and 2012 have been conducted. Cosmic ray data were obtained from the KACST muon detector in Riyadh, Saudi Arabia ( 24 43; 46 40; Rc =14.4 GV). We have found that cosmic ray muons are anti-correlated with both parameters. The same analyses have been carried out using data from the Oulu neutron monitor and comparable results with the KACST detector have been found.
\end{abstract}




\section{Introduction}

Instabilities of the Sun affect interplanetary space and heliosphere. The results of these solar disturbances are the modulation of galactic cosmic rays at different time scales [1]. There are two kinds of intensity variations: one is sporadic, such as Forbush decreases, and the other is periodic variations, on timescales of either short-term (e.g. 27 days) or long-term (e.g. 11 years) variations [2-3].

The relationship between solar activity parameters and variations of cosmic ray intensity has been studied by several investigators [e.g., 4]. Different activity parameters have been used to quantify the effect of solar activity on cosmic ray particles. Several investigators consider the sunspot numbers as a reliable measure of this activity [5], while others used the solar wind plasma speed or the interplanetary magnetic field [6-8]. Sun spot numbers are generally considered to be one of the more representative and obtainable indicators of solar activity [1314]. On the other hand, solar wind is magnetised plasma that generates continuous flow from the sun with different amounts. Due to the associated magnetic field, solar wind affects the incoming charged particles [9].

In this study, the impact of some solar activity parameters on the cosmic ray data is studied quantitatively. This is achieved by conducting correlative analysis between solar wind speed (V) and smoothed sunspot number (SSN) and the cosmic ray data from the King Abdulaziz City for Science and Technology (KACST) muon detector and Oulu neutron monitor for the period between 2002 and 2012. In the following sections, the observational data and methodology will be briefly described, followed by a discussion of the obtained results and associated interpretations.

\section{Method of analysis}

The KACST detector is located in Riyadh in central Saudi Arabia (Lat. 24 43; Long. 46 40). The location of the detector is considered a unique place for cosmic ray studies due its high cutoff rigidity $(14.4 \mathrm{GV})$. The detector is a $1 \mathrm{~m}^{2}$ plastic scintillator viewed by a photomultiplier tube (PMT). The PMT detects the flash of light produced when cosmic ray muons interact with the scintillator materials. The signals from the PMT are amplified and digitised by a homemade analogue to digital converter (ADC).

The detector was been in operation since July 2002, and data collected from that time until August 2012 are used in this study. This period covers the declining phase of solar cycle 23 and beginning of the 24th solar cycle. The detector and its specifications are detailed in[10-11].For comparison purposes, the pressure-corrected cosmic ray data from the Oulu neutron monitor collected over the same time period are also used in this study.

Solar wind plasma speeds for the considered period were obtained from NASA's Omni website (http://omniweb.gsfc.nasa.gov/form/dx1.html). The new version (V2) of SSN were collected from the WDC-SILSO of the Royal Observatory of Belgium in Brussels[12]. The new version, which was updated in 2015, has several corrections to account for inhomogeneities found in the time series of the old version. The correction procedures and details of this new version are given in several references [12-14]. The V2 sunspots were smoothed using the approach discussed in [15] and their mean monthly values along with the solar wind speed and 
On the relationship between cosmic rays detected by the KACST muon detector and solar wind velocity and sunspot Number

muon data between 2002 and 2012 were used. Raw muon data from the KACST detector have been corrected for the pressure effect using the method described in [16-17]

\section{Results and discussion}

Figure (1) is a long-term plot of the mean monthly values of cosmic ray muons from the KACST detector, and neutrons from the Oulu station. It can be seen that the number of cosmic rays from both detectors increases to a maximum in 2009, in which the data from the neutron monitor are smoother than those from the muon detector. This may be explained by the fact that cosmic ray muons are more sensitive to seasonal and atmospheric variations than cosmic ray neutrons [17].

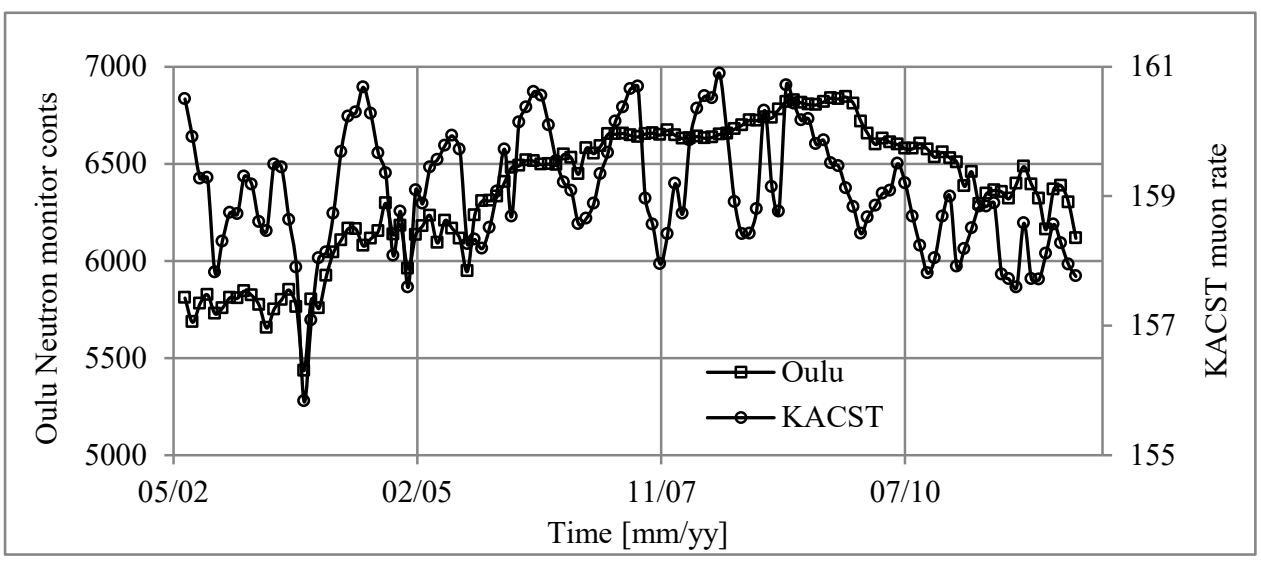

Figure (1) shows the interannual variations of the mean monthly values of the cosmic ray muons from the KACST detector and neutrons from the Oulu station.

Figure (2) displays the long-term data for the mean monthly V and smoothed SSN. The SSN decrease gradually from about 160 in 2002 to a minimum of about 3 in 2009. They then resume an increase afterward. Apart from the increase between 2006 and 2007, V exhibited a decreasing trend and reaches a minimum in 2009.

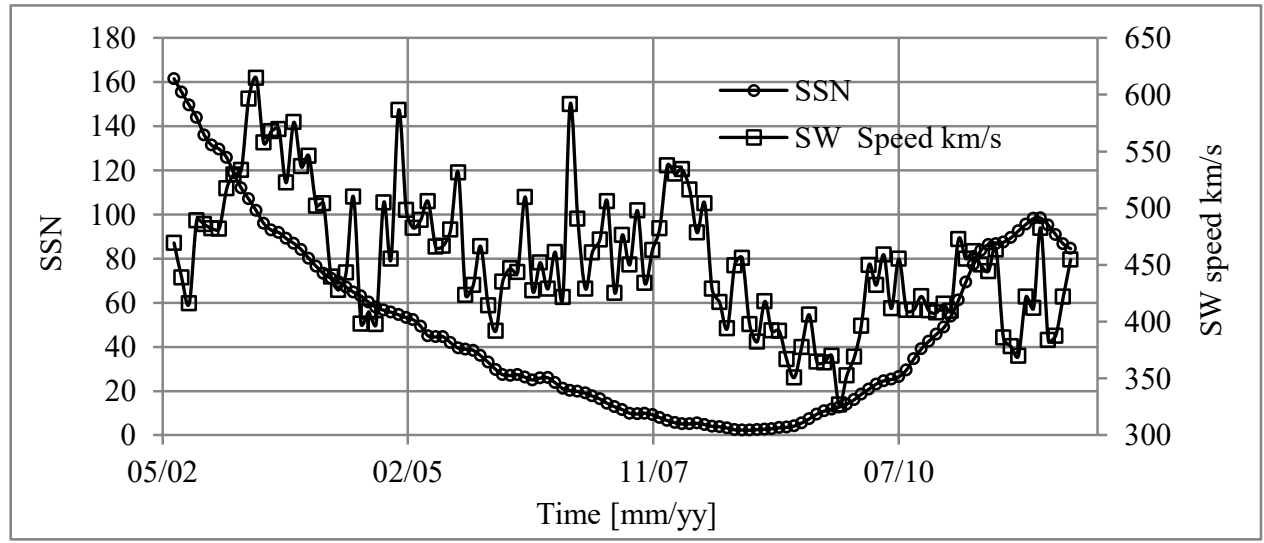

Figure (2) shows the interannual variations of the mean monthly values of unspot number (SSN) and solar wind (SW) speed, between 2002-2012. 

and sunspot Number

Figure (3) is a scatter plot that shows the relationship between the smoothed SSN and cosmic rays from the (a) KACST detector and (b) Oulu neutron monitor. Evidently, the cosmic rays from both data sets are anti-correlated with the SSN. Nevertheless, the relationship is more obvious for the neutron data than for the muons. Regression analyses show that the muon rate decreases by $0.012 \pm 0.001 \%$ with an increase of one SSN with a correlation coefficient of 0.54 . Similarly, the neutrons decrease by $0.11 \pm 0.02 \%$ per one sunspot with a correlation of 0.83 .
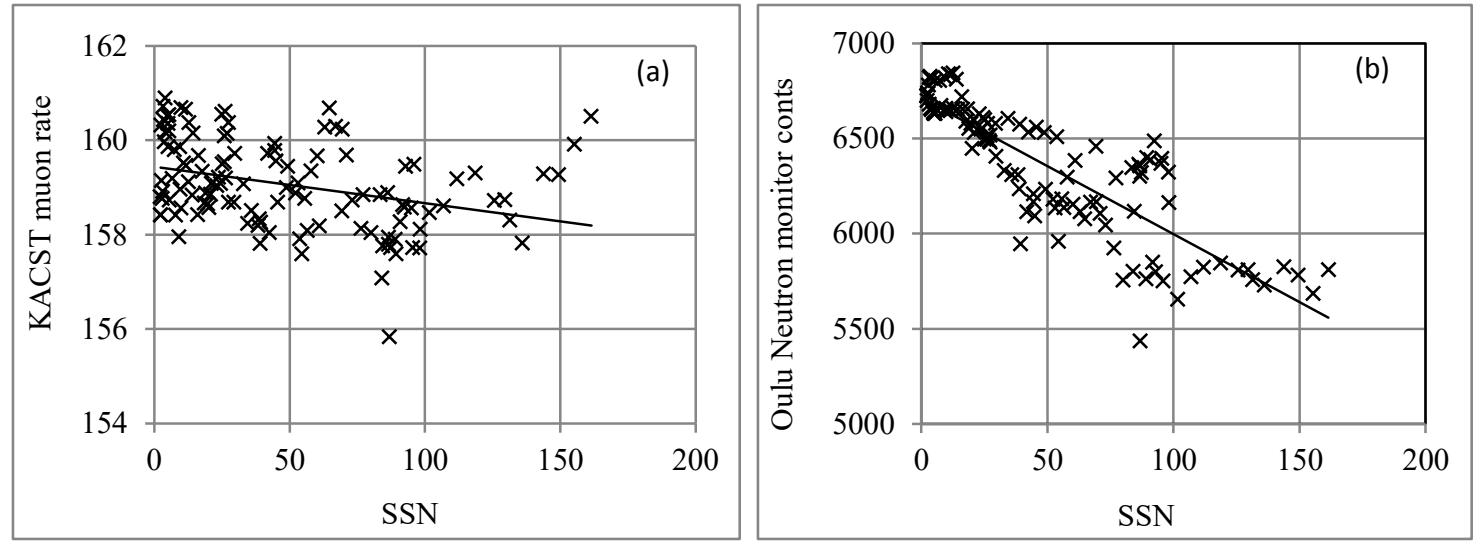

Figure (3) displays the relationship between the smoothed SSN and cosmic rays from (a) muons from the KACST detector and (b) the Oulu neutron monitor. The straight line is the best linear fit to the data.

Figure (4) presents the relationship between $\mathrm{V}$ and the (a) muon rates and (b) neutron counts. The scatter in the data may be due to inhomogeneities in the amount of solar wind in different solar activity phases[9]. In the case of the muon particles, the rate decreases by $0.0029 \pm 0.0004 \% / \mathrm{km} / \mathrm{s}$ with a correlation coefficient of 0.5 . The neutron monitor data show a decrease by $0.05 \pm 0.002 \% / \mathrm{km} / \mathrm{s}$ with a correlation of 0.83 . These results suggest there is clear opposite relationship between $\mathrm{V}$ and the cosmic ray intensity. The strengths of these correlations are stronger for cosmic ray neutrons than for muons. The differences in rigidity and the type of the detector may cause some of these differences. Additionally, the atmospheric, meteorological and extraterrestrial variations, which have more influence on the cosmic ray muons than the neutrons, may be considered a possible reason for these differences in dependence[17 - 18]. The obtained cofficients are in close agreements with those obtained by several investigators. For instance, [8] showed a decrease of 0.001 for the muon data recorded by the GRABE muon detector and 0.003 for the neutron monitor data from the Kiel station. 
On the relationship between cosmic rays detected by the KACST muon detector and solar wind velocity and sunspot Number
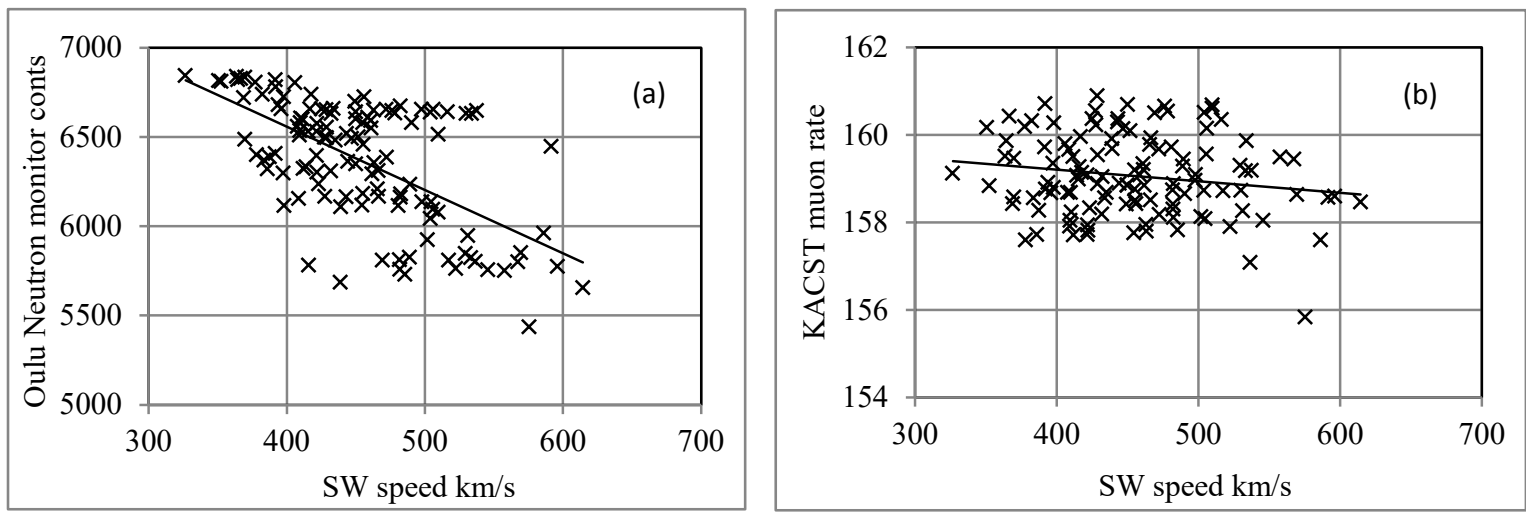

Figure (4) displays the relationship between V and cosmic rays from (a) muons from the KACST detector and (b) the Oulu neutron monitor. The straight line is the best linear fit to the data.

In order to obtain a quantitative estimate of the above results, the monthly data for all considered parameters have been categorised into six groups according to V: less than $350 \mathrm{~km} /$ $\mathrm{s} ; 351-400 \mathrm{~km} / \mathrm{s} ; 401-450 \mathrm{~km} / \mathrm{s} ; 451-500 \mathrm{~km} / \mathrm{s} ; 501-550 \mathrm{~km} / \mathrm{s}$ and greater than $551 \mathrm{~km} / \mathrm{s}$. The results presented in Figure (5) demonstrate clear and strong correlations between cosmic ray intensities for the two cosmic ray components with the two parameters.
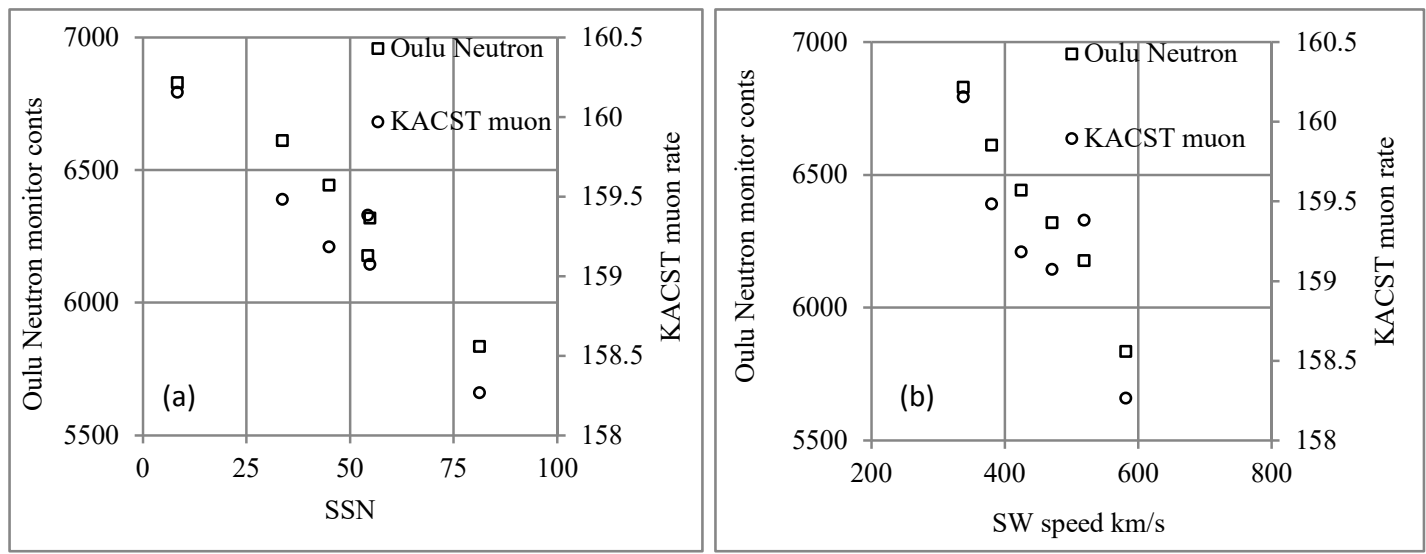

Figure (5) displays the relationship between the categorised (a) V and (b) SSN values and the cosmic rays from the KACST detector and Oulu neutron monitor.

For the muon data, the regression analyses give a sunspot coefficient of $-0.015 \pm 0.003 \%$ per every increase in the SSN with a correlation coefficient $=0.95$, and a solar wind coefficient of $0.0037 \pm 0.0006 \% / \mathrm{km} / \mathrm{s}$ with a correlation coefficient of 087 . For the data from the Oulu monitor, on the other hand, it was found that the number of cosmic ray neutrons decreases by $0.21 \pm 0.02 \%$ for every increase in the SSN and $0.05 \pm 0.001 \% /$ and $\mathrm{km} / \mathrm{s}$. The correlation coefficient for both cases was greater than 0.95. Although the effect of the considered parameters on the detected cosmic ray particles is obvious, the obtained results from the categorised data indicate better correlations than those obtained from the entire data set. The obtained proportionality coefficients did not differ much between the two data sets. 
On the relationship between cosmic rays detected by the KACST muon detector and solar wind velocity and sunspot Number

\section{Conclusion}

In this study, monthly mean values of solar wind speed and sunspot number have been used to study their influence on secondary cosmic ray particles. Cosmic ray data for the period between 2002 and 2012 from the KACST muon detector and Oulu neutron monitor have been utlized for the purpose of this study. We find that the cosmic ray muons and neutrons are anticorrelated with these two parameters. The strengths of these correlations are stronger for cosmic ray neutrons than for muons. Clearer correlations are found when the data are categorised according to solar wind speed. The obtained coefficients for the effect of the sunspot number and solar wind speed on the cosmic ray muons and neutrons are within the range of the previously established values.

\section{Acknowledgments}

The authors would like to thank King Abdulaziz City for Science and Technology (KACST) for supporting this work.

\section{References}

[1] K. Kudela, 2016. On low energy cosmic rays and energetic particles near Earth, Contrib. Astron. Obs. Skalnate Pleso, 46, 15-70, 2016.

[2] H. Cane et al., Cosmic ray modulation and the solar magnetic field. Geophys. Res., Lett. 26, $565-$ $568,1999$.

[3] P Kane, Long term variations of solar interplanetary geomagnetic indices and Cosmic ray intensities. Indian J Radio \& Space Physics, 35:312, 2006.

[4] S. Gupta, et al., Correlative analysis of long-term cosmic ray modulation with solar activity parameters Indian J.Sci.Res.2(4) 1-14, 2011.

[5] M. Gupta, et al., Study of Cosmic ray intensity variations in relation to solar activity for Sunspot cycles 19 to 23. Indian J. Radio \& Space Physics, 35:167, 2006.

[6] K. Fujimoto, et al., Cosmic Ray Intensity Variations and Solar Wind Velocity, 18th ICRC, Bangalore, 3, 267, 1983

[7] K. Fujimoto, et al., The Solar Wind Effect on Cosmic Rays and the Solar Activity, 19th ICRC, San Diego, 3, 262, 1985.

[8] H. Kojima et al., Dependence of cosmic ray intensity on variation of solar wind velocity measured by the GRAPES-3 experiment for space weather studies, PHYSICAL REVIEW D 91, 121303(R) 2015.

[9] V. Dwivedi, et al., Solar wind speed variations on other interplanetary parameters. Indian J. Radio \& Space Physics, 39:252, 2010.

[10] Maghrabi A. et al., The KACST muon detector and its application to cosmic-ray variations studies, Advances in Space Research, 50, 6,700-711, 2012.

[11] Maghrab A. et al., On the calibration of a single channel cosmic ray particle detector, Proc. SPIE 9154, VI, 91542K, 2014. 
On the relationship between cosmic rays detected by the KACST muon detector and solar wind velocity and sunspot Number

[12] F.Clette, Lefèvre, L., The New Sunspot Number: assembling all corrections, Solar Physics, 291, DOI: 10.1007/s11207-016-1014-y, 2016.

[13] F Clette, et al., 2016: The Revised Brussels-Locarno Sunspot Number (1981 - 2015), Solar Physics, 291, 2016.

[14] F. Clette, et al., 2016: Preface to Topical Issue: Recalibration of the Sunspot Number, Solar Phys., 291, 2016.

[15] A McNish, Lincoln J V. Prediction of sunspot numbers. Trans. Am. Geophys. Union, 30: 673-685, 1949.

[16] A Maghrabi. Et al., Influence of the Atmospheric Mass on the High Energy Cosmic Ray Muons during a Solar Cycle, Advances in Astronomy, Vol.2015 ,ID939146-2015.

[17] Maghrabi A.H., Almutayri M., Atmospheric Effect on Cosmic Ray muons at High Cutoff Rigidity Station, Advances in Astronomy, V. 2016, Article ID 9620189, 2016,

[18] Bchelet, F., et al., The cosmic-ray spectral modulation above 2 GV, Nuovo Cimento 11B, 1-12, 1972. 